\title{
Letters:
}

\section{Primary pulmonary rhabdomyosarcoma in an adult: a case report and review of the literature}

\author{
Gui-yi JI, Hui MAO ${ }^{\dagger *}$ \\ (Department of Respiratory Medicine, West China Hospital of Sichuan \\ University, Chengdu 610041, China) \\ †E-mail: merrymh@126.com \\ Received Dec. 13, 2012; Revision accepted Apr. 1, 2013 \\ Crosschecked Aug. 13, 2013
}

doi:10.1631/jzus.B1200248

Document code: A

CLC number: R445; R734.2

Primary pulmonary rhabdomyosarcoma (RMS) is one of the most common soft-tissue malignancies in children, but is rare in persons aged $>45$ years (Triche, 1997; Ferrari et al., 2003). RMS is considered to result from the malignant transformation of primitive mesenchymal cells. The head, neck, and limbs are the principal locations of RMS. Primary pulmonary RMS is extremely rare and is frequently misdiagnosed as small cell lung cancer.

In this letter, we report a case of primary pulmonary RMS in an adult patient, which was the only histologically confirmed case of RMS diagnosed between January 1995 and July 2012 in West China hospital. A 51-year-old man presented with a dull pain in the left chest, tightness of breath for one month, and aggravation of these symptoms for $2 \mathrm{~d}$. A soft-tissue mass in the upper left lung was detected on chest computed tomography (CT). Fiberoptic bronchoscopy showed a cauliflower-like neoplasm in the lower segment of the left main bronchus. Immunohistochemical examination of a biopsy specimen showed that the neoplasm was an RMS, as the cy-

\footnotetext{
${ }^{\ddagger}$ Corresponding author

* Project supported by the National Natural Science Foundation of China (Nos. 30600265, 30871119, and 81070024), the Scientific Research Foundation for the Returned Overseas Chinese Scholars, Ministry of Education of China, and the State Administration of Traditional Chinese Medicine of China (No. JDZX2012032)

(C) Zhejiang University and Springer-Verlag Berlin Heidelberg 2013
}

toplasm of the tumor cells was diffusely positive for desmin, and the nuclei were partially positive for myogenin. Some tumor cells showed polyploid chromosomes. Although three cycles of chemotherapy were administered, the patient died 8 months after the diagnosis because of tumor deterioration. Currently, no effective therapeutic strategies for RMS exist. Further studies are required to analyze the prognostic factors, histological features, and molecular biological characteristics of RMS, which remain controversial or unknown.

A 51-year-old man visited our respiratory department with chief complaints of dull pain in the left chest and tightness of breath since 1 month, and aggravation of these symptoms since $2 \mathrm{~d}$. The chest $\mathrm{CT}$ scan showed a space-occupying lesion in the left main bronchus. An enhanced chest CT scan revealed complete atelectasis of the left lung due to obstruction of the left main bronchus by a soft-tissue mass; the mediastinum was shifted to the left (Fig. 1a). Fiberoptic bronchoscopy showed a cauliflower-like neoplasm in the lower segment of the left main bronchus (Fig. 2a). Biopsy examination of the mass suggested a small cell carcinoma; however, the results were inconclusive. We therefore repeated the fiberoptic bronchoscopy and resected a part of the mass in the left main bronchus (Fig. 2b). Immunohistochemical examination showed that the neoplasm was an RMS, as the cytoplasm of the tumor cells was diffusely positive for desmin and the nuclei were partially positive for myogenin (Fig. 3) (Pappo et al., 1995; Dias et al., 2000). The FOXO1 (FKHR) gene translocation was not detected on fluorescent in situ hybridization. Some tumor cells showed polyploid chromosomes. A repeat chest CT scan (Fig. 1b) showed an $8.6 \mathrm{~cm} \times$ $7.2 \mathrm{~cm}$, lobulated, soft-tissue mass in the upper left lung near the hilum. Magnetic resonance imaging showed multiple cysts and small nodules in the liver, 
necessitating further investigation to determine whether these were metastases. A bone scan and bone marrow biopsy ruled out bone involvement. The final diagnosis was central-type, alveolar RMS (ARMS) of the left lung (cT3NxM0, stage III). Surgery was not indicated, and the patient was referred to the Oncology Department for Chemotherapy. He was administered a multidrug regimen that included ifosfamide (2.0 g, Days 1-2, intravenous intravenously guttae (ivgtt)), epirubicin (80 mg, Day 1, iv), and dacarbazine (200 mg, Days 1-3, ivgtt). After two cycles of chemotherapy, his symptoms were relieved, and chest CT showed that the lesion in the left lung was slightly smaller than it had been 2 months ago (Fig. 1c). One more cycle of chemotherapy was administered, but its effects were very limited. The final chest CT showed that the tumor was significantly larger than it had been 8 months ago (Fig. 1d). The patient died because (a)

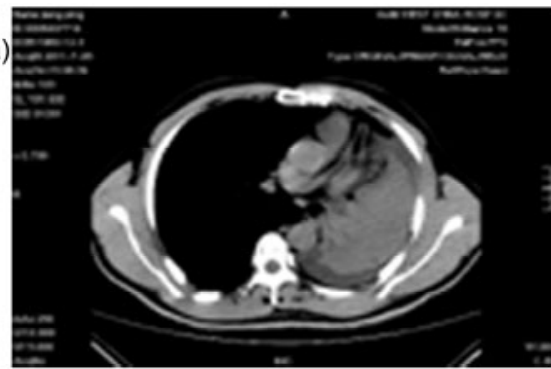

(c)

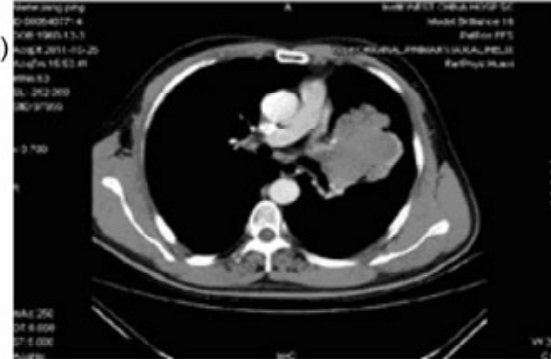

(b)

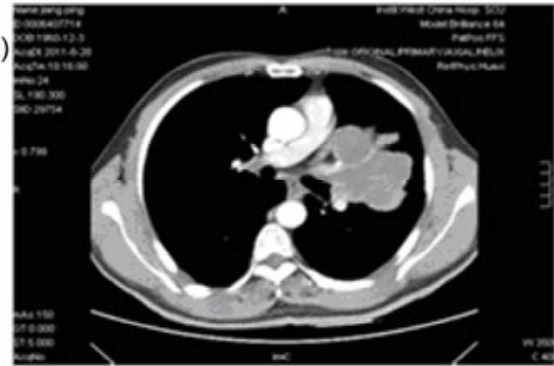

(d)

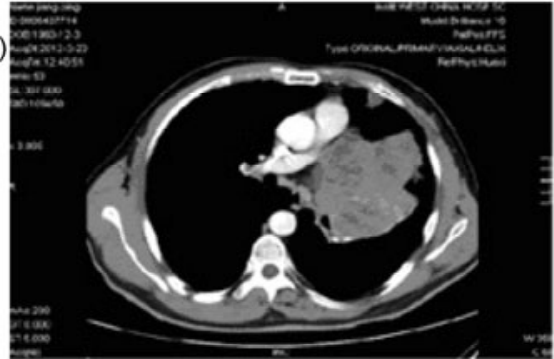

Fig. 1 Chest computed tomography (CT) scans of the patient during different periods

(a) July 28, 2011, the chest CT scan shows complete atelectasis of the left lung due to obstruction of the left main bronchus by a soft-tissue mass; (b) Aug. 28, 2011, after a second fiberoptic bronchoscopy, the chest CT scan shows a lobulated soft-tissue mass $(8.6 \mathrm{~cm} \times 7.2 \mathrm{~cm})$ in the upper left lung near the hilum; (c) Oct. 26, 2011, after two cycles of chemotherapy, the chest CT scan shows that the lesion in the left lung is slightly smaller; (d) Mar. 23, 2012, after three cycles of chemotherapy, the chest CT scan shows that the tumor has significantly enlarged

(a)

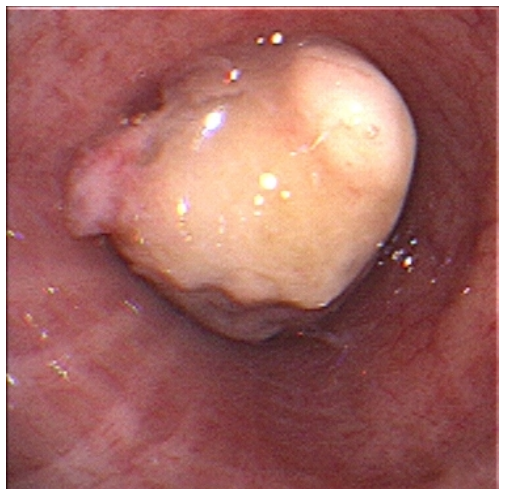

(b)

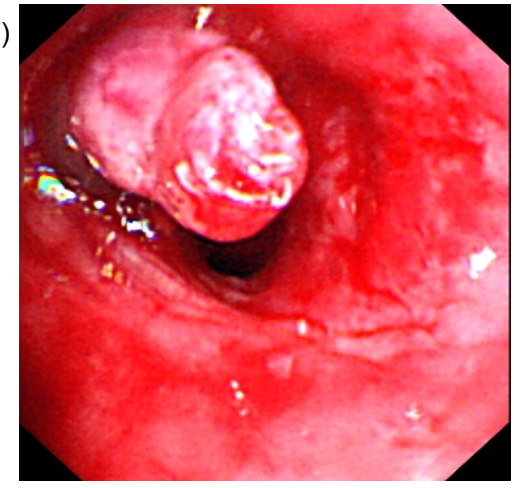

Fig. 2 Bronchoscopic images of the patient

(a) Bronchoscopic images (July 27, 2011) showing a cauliflower-like neoplasm in the lower segment of the left main bronchus. The lesion has completely blocked the lumen of the bronchus, and bronchoscope cannot be passed further. (b) Bronchoscopy was repeated on Aug. 3, 2011 and part of the tumor mass was removed. The tumor can be seen to originate from the upper left lobe bronchus; the left lower lobe bronchus openings are not involved 
(a)

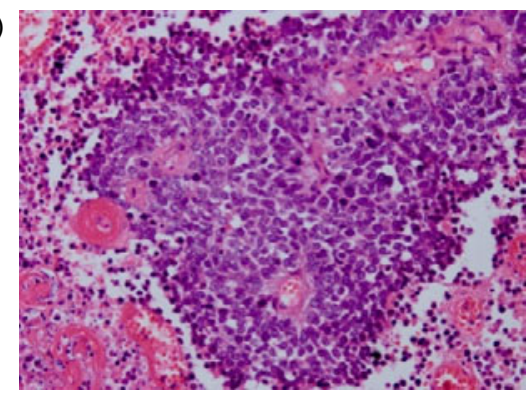

(b)

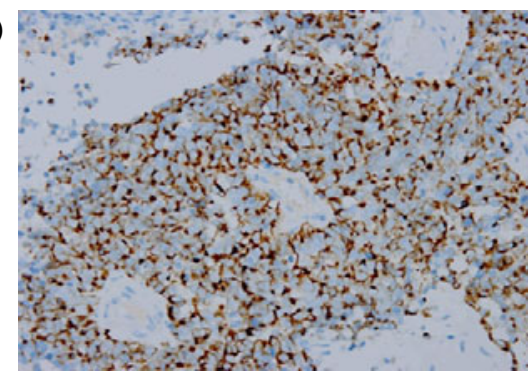

(c)

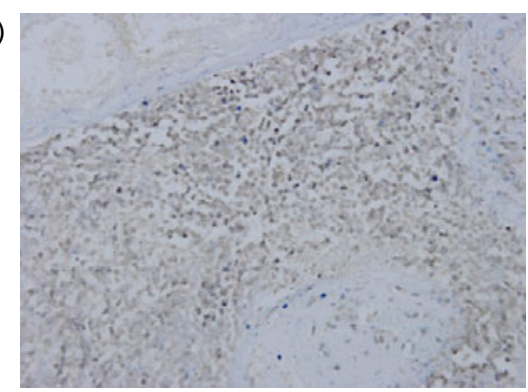

Fig. 3 Hematoxylin-eosin (HE) staining and immunohistochemical staining $(400 \times)$

(a) HE staining of a bronchial biopsy specimen showing scattered or clustered tumor cells, with round or oval basophilic nuclei and marked atypia; (b) Cytoplasmic desmin expression is detected in most tumor cells on immunohistochemical staining; (c) Nuclear myogenin expression is detected in some tumor cells on immunohistochemical staining

of tumor deterioration in June 2012. This study was approved by the Human Ethics Committee of Sichuan University and performed in accordance with the Guidelines for Human Experimentation of Sichuan University.

RMS is one of the most common mesenchymal malignancies in children and adolescents (Ferrari et al., 2003), accounting for $5 \%-15 \%$ of all solid malignancies and $4 \%-8 \%$ of all malignancies in children aged under 15 years (Koumarianou et al., 2012). RMS is extremely rare in individuals aged more than 45 years. RMS results from the biological changes caused by aberrations in the transcription signals that lead to myogenic protein production (Koumarianou et al., 2012). This tumor possibly originates from pluripotent cells, which can differentiate into muscle cells. RMS can occur in various locations, but primary RMS is rarely found in the lungs, which contain no striated muscle (Parham and Ellison, 2006); rather, the lung is the most common organ for tumor metastasis. RMS causes local symptoms related to mass lesions, infiltration and destruction of adjacent tissues (Parham and Ellison, 2006). It has diverse, nonspecific clinical manifestations and is therefore easily misdiagnosed.

RMS can be divided into embryonal and alveolar subtypes based on its pathological features (Sorensen et al., 2002). Recently, the RMS classification was expanded to include the botryoid and spindle cell variants of the embryonal subtype, and a rare primitive tumor termed undifferentiated sarcoma (Newton et al., 1995; Sorensen et al., 2002). The clinical classification of RMS is based on local and distant spread and the possibility of surgical removal of the local lesion; primary tumor sites are usually classified as favorable or less-favorable (Wexler and Helman, 1997; Sorensen et al., 2002). Owing to the great variation in RMS outcomes, many researches have been focused on determining prognostic indicators that can be used for therapeutic stratification of RMS patients (Crist et al., 1990; Sorensen et al., 2002). Currently, the most useful prognostic indicators are extent of disease at presentation, site of primary tumor, histological characteristics, and type of therapy administered (Crist et al., 1990). Detailed classifications of RMS are helpful to evaluate patient prognosis (Triche, 1997; Parham and Ellison, 2006). In general, botryoid and spindle cell RMS have a good prognosis, embryonal RMS (ERMS) has an intermediate prognosis, and ARMS has a poor prognosis (Parham and Ellison, 2006). ERMS principally presents in areas with little skeletal muscle, including the head and neck, retroperitoneum and genitourinary tract, in children aged $<10$ years and accounts for approximately $60 \%$ of all RMS cases. The 5 -year survival rate of ERMS is $70 \%$. In contrast, ARMS often involves sites that are adjacent to skeletal muscle, in the extremities and trunk. It primarily affects adolescents and young adults, and accounts for approximately $20 \%$ of all cases of RMS. The 5 -year survival rate of ARMS is $<10 \%$. 
The mechanisms underlying the development of RMS remain unclear. Analysis of recurrent cytogenetic alterations in solid tumors has shown that reciprocal chromosomal translocations are related to sarcomas (Sorensen et al., 2002). Cloning studies have demonstrated in-frame fusion of the coding sequences of rearranged genes, resulting in fusion transcripts that encode oncogenic chimeric proteins with oncogenic activity (Sorensen et al., 2002). The detection of chromosome translocation and fusion genes can facilitate the diagnosis and classification of RMS. Chromosomal analyses have demonstrated that the translocations $\mathrm{t}(2 ; 13)(\mathrm{q} 35 ; \mathrm{q} 14)$ and $\mathrm{t}(1 ; 13)(\mathrm{p} 36$; q14) are associated with ARMS (Davis et al., 1994; Fredericks et al., 1995; Ladanyi, 1995; Sorensen and Triche, 1996; Sorensen et al., 2002). Some studies demonstrated that these translocations fuse the forkhead box O1 (FKHR) locus on chromosome 13 to either PAX3 on chromosome 2 or PAX7 on chromosome 1 (Galili et al., 1993; Folpe et al., 2002; Sorensen et al., 2002). The $\mathrm{t}(2 ; 13)(\mathrm{q} 35-37 ; \mathrm{q} 14)$ translocation, which generates the PAX3-FKHR chimeric gene, has been identified in $70 \%$ of ARMS patients. In addition, a variant translocation, $\mathrm{t}(1 ; 13)(\mathrm{p} 36 ; \mathrm{q} 14)$, which generates the $P A X 7-F K H R$ chimeric gene, has been detected in $<20 \%$ of ARMS patients. These two translocations are sensitive and specific markers for ARMS and have not been found in any other tumor (Triche, 1997). The FKHR gene has been detected in $80 \%$ of ARMS patients. In ERMS, a consistent loss of heterozygosity $(\mathrm{LOH})$ at $11 \mathrm{p} 15$ is detectable (Besnard-Guerin et al., 1996; Chang et al., 1996).

Developing muscle expresses unique transcription factors that initiate myogenesis (Barr, 2001); therefore, muscle cells have a relatively unique phenotype. The markers desmin and myogenin are useful in the diagnostic work-up of ARMS (Parham and Ellison, 2006). Desmin is also found in other small round cell tumors, especially desmoplastic small round cell tumor (Ordonez, 1998). Therefore, desmin is not a specific marker of ARMS, but forms a useful part of a diagnostic panel for ARMS. The transcription factors myogenic determination factor (MyoD) and myogenin are highly sensitive and specific for RMS (Cessna et al., 2001). In general, ARMS cells show nuclear expression of MyoD and myogenin (Dias et al., 2000), whereas ERMS cells stain in a more heterogeneous fashion. Unlike other forms of
ARMS, sclerosing RMS, a newly described variant, is strongly positive for MyoD, but only weakly positive for myogenin (Folpe et al., 2002).

In our patient, the tumor was misdiagnosed as a small cell lung cancer on pathological examination of the biopsy specimen obtained using the first fiberoptic bronchoscopy. However, immunohistochemical examination of a second biopsy specimen, obtained by repeating the bronchoscopy, showed that the cytoplasm of the tumor cells was diffusely positive for desmin, while the nuclei were partially positive for myogenin. These results indicated that the tumor was an RMS. The diagnosis of RMS depends on comprehensive analysis of data from clinical, histological, immunohistochemical, electron microscopic, and molecular biological examinations (Gurney et al., 2005). In the past, RMS was mainly diagnosed using electron microscopy. With the development of genetic tests and immunohistochemistry, the use of electron microscopy for the diagnosis of RMS has declined. Currently, immunohistochemistry and molecular biology are particularly important for the diagnosis of RMS (Parham and Ellison, 2006). The identification of myogenic markers, and in particular, microchip analysis, can help identify RMS in cases of diagnostic difficulty (Golub et al., 1999).

To our knowledge, no therapeutic regimen for RMS has proved absolutely effective. RMS treatments are selected according to the clinical TNM classification (Table 1) (Koumarianou et al., 2012), the pathological subtype, and the intergroup rhabdomyosarcoma study (IRS) post-surgical grouping system (Table 2) (Koumarianou et al., 2012). It was reported that the tumor mass decreased after induction chemotherapy in $77 \%$ of RMS patients, the majority of whom showed only a partial response before planned local control (Burke et al., 2007; Minn et al., 2010). Unless patients exhibit disease progression, induction of chemotherapy, local control with radiation therapy, surgery or both, and chemotherapy should be continued as planned (Minn et al., 2010). Our patient had advanced disease, and we therefore administered only chemotherapy. The patient refused further radiation therapy, and did not receive treatment for local control. Although chemotherapy can help to some extent in the early stages, RMS, being a malignant tumor, carries a poor prognosis. Metastasis occurs in most RMS patients, leading to inoperability. 
Table 1 TNM classification for rhabdomyosarcoma (RMS) (Koumarianou et al., 2012)

\begin{tabular}{|c|c|c|c|c|}
\hline Stage & Site of primary tumor & Tumor size & Regional lymph node & Distant metastases \\
\hline 1 & $\begin{array}{l}\text { Orbit, non-parameningeal head and neck, } \\
\text { genitourina non-bladder/prostate, biliary tract }\end{array}$ & Any size & $\mathrm{N} 0, \mathrm{~N} 1$ & M0 \\
\hline 2 & All other sites & $\leq 5 \mathrm{~cm}$ & No & M0 \\
\hline 3 & All other sites & $\begin{array}{l}\leq 5 \mathrm{~cm} ; \\
>5 \mathrm{~cm}\end{array}$ & $\begin{array}{c}\text { N1; } \\
\text { N0, N1 }\end{array}$ & M0 \\
\hline 4 & Any sites & Any size & N0, N1 & M1 \\
\hline
\end{tabular}

N0: regional nodes not clinically involved; N1: regional nodes clinically involved by neoplasm; M0: no distant metastasis; M1: metastasis present

Table 2 Classification of rhabdomyosarcoma (RMS) into four post-surgical pathologic groups based on the extent of the residual disease after initial surgical treatment (Koumarianou et al., 2012)

\begin{tabular}{|c|c|}
\hline Group & Definition \\
\hline I & $\begin{array}{l}\text { Localized tumor, completely removed with pathologically clear margins and no regional lymph node } \\
\text { involvement }\end{array}$ \\
\hline II & $\begin{array}{l}\text { Localized tumor, grossly removed with (a) microscopically involved margins, (b) involved, grossly } \\
\text { resected regional lymph nodes }\end{array}$ \\
\hline III & Localized tumor, with gross residual disease after grossly incomplete removal, or biopsy only \\
\hline IV & Distant metastases present at diagnosis \\
\hline
\end{tabular}

While advances in molecular biology have offered novel therapeutic strategies, the treatment of RMS remains a clinical challenge.

As primary pulmonary RMS is extremely rare, we could not find any treatment recommendations for this condition. Single-agent therapy with antineoplastic drugs such as vincristine, dactinomycin, and cyclophosphamide was shown to be effective against RMS in the early 1960s (Crist et al., 2001). In the 1970s, combination chemotherapy (vincristine, dactinomycin, and cyclophosphamide [VAC regimen]) along with radiation therapy was shown to increase complete response rates among newly diagnosed patients. In a recent study of pediatric RMS, Crist et al. (2001) reported that the VAC regimen, with or without radiation treatment, was the gold standard for RMS. However, none of the regimens they compared was superior to the others. Moreover, this therapeutic gain was negatively offset by a slightly worse outcome for patients with ARMS or undifferentiated RMS. A new therapeutic approach is required for these patients. Topotecan is the most promising new agent for RMS. Clinical studies have indicated that topotecan is highly active against human RMS, especially, ARMS (Houghton et al., 1991; Crist et al., 2001; Pappo et al., 2001).

In summary, primary pulmonary RMS is rare and can be easily misdiagnosed as small cell lung cancer. The diagnosis of RMS depends mainly on pathologic, immunohistochemical, and molecular biological studies. Currently, no effective therapeutic regimens for RMS exist. Further studies are required to analyze the prognostic factors, histological features, and molecular biological characteristics of RMS, which are either controversial or unknown.

\section{Compliance with ethics guidelines}

Gui-yi JI and Hui MAO declare that they have no conflict of interest.

All procedures followed were in accordance with the ethical standards of the responsible committee on human experimentation (institutional and national) and with the Helsinki Declaration of 1975 , as revised in 2000(5). Informed consent was obtained from all patients for being included in the study.

Additional informed consent was obtained from all patients for which identifying information is included in this article.

\section{References}

Barr, F.G., 2001. Gene fusions involving PAX and FOX family members in alveolar rhabdomyosarcoma. Oncogene, 20(40):5736-5746. [doi:10.1038/sj.onc.1204599]

Besnard-Guerin, C., Newsham, I., Winquist, R., Cavenee, W.K., 1996. A common region of loss of heterozygosity 
in Wilms' tumor and embryonal rhabdomyosarcoma distal to the D11S988 locus on chromosome 11p15.5. Hum. Genet., 97(2):163-170. [doi:10.1007/BF02265259]

Burke, M., Anderson, J.R., Kao, S.C., Rodeberg, D., Qualman, S.J., Wolden, S.L., Meyer, W.H., Breitfeld, P.P., 2007. Assessment of response to induction therapy and its influence on 5-year failure-free survival in group III rhabdomyosarcoma: the intergroup rhabdomyosarcoma studyIV experience-a report from the Soft Tissue Sarcoma Committee of the Children's Oncology Group. J. Clin. Oncol., 25(31):4909-4913. [doi:10.1200/JCO.2006.10. 4257]

Cessna, M.H., Zhou, H., Perkins, S.L., Tripp, S.R., Layfield, L., Daines, C., Coffin, C.M., 2001. Are myogenin and MyoD1 expression specific for rhabdomyosarcoma? A study of 150 cases, with emphasis on spindle cell mimics. Am. J. Surg. Pathol., 25(9):1150-1157. [doi:10.1097/0000 0478-200109000-00005]

Chang, W.Y., Yuan, L., Feng, L., Hensle, T., Tycko, B., 1996. Chromosome 11p15.5 regional imprinting: comparative analysis of KIP2 and H19 in human tissues and Wilms' tumors. Hum. Mol. Genet., 5(8):1101-1108. [doi:10. 1093/hmg/5.8.1101]

Crist, W.M., Garnsey, L., Beltangady, M.S., Gehan, E., Ruymann, F., Webber, B., Hays, D.M., Wharam, M., Maurer, H.M., 1990. Prognosis in children with rhabdomyosarcoma: a report of the intergroup rhabdomyosarcoma studies I and II-Intergroup Rhabdomyosarcoma Committee. J. Clin. Oncol., 8(3):443-452.

Crist, W.M., Anderson, J.R., Meza, J.L., Fryer, C., Raney, R.B., Ruymann, F.B., Breneman, J., Qualman, S.J., Wiener, E., Wharam, M., et al., 2001. Intergroup rhabdomyosarcoma study-IV: results for patients with nonmetastatic disease. J. Clin. Oncol., 19(12):3091-3102.

Davis, R.J., D'Cruz, C.M., Lovell, M.A., Biegel, J.A., Barr, F.G., 1994. Fusion of PAX7 to FKHR by the variant $\mathrm{t}(1 ; 13)(\mathrm{p} 36 ; \mathrm{q} 14)$ translocation in alveolar rhabdomyosarcoma. Cancer Res., 54(11):2869-2872.

Dias, P., Chen, B., Dilday, B., Palmer, H., Hosoi, H., Singh, S., Wu, C., Li, X., Thompson, J., Parham, D., et al., 2000. Strong immunostaining for myogenin in rhabdomyosarcoma is significantly associated with tumors of the alveolar subclass. Am. J. Pathol., 156(2):399-408. [doi:10. 1016/S0002-9440(10)64743-8]

Ferrari, A., Dileo, P., Casanova, M., Bertuli, R., Gandola, L., Navarria, P., Collini, P., Cronchi, A., Olmi, P., FossatiBellani, F., et al., 2003. Rhabdomyosarcoma in adults. A retrospective analysis of 171 patients treated at a single institution. J. Cancer, 98(3):571-580. [doi:10.1002/cnr. 11550]

Folpe, A.L., McKenney, J.K., Bridge, J.A., Weiss, S.W., 2002. Sclerosing rhabdomyosarcoma in adults: report of four cases of a hyalinizing, matrix-rich variant of rhabdomyosarcoma that may be confused with osteosarcoma, chondrosarcoma, or angiosarcoma. Am. J. Surg. Pathol., 26(9):1175-1183. [doi:10.1097/01.PAS.0000024996.88 486.CA]
Fredericks, W.J., Galili, N., Mukhopadhyay, S., Rovera, G., Bennicelli, J., Barr, F.G., Rauscher, F.J., 1995. The PAX3FKHR fusion protein created by the $\mathrm{t}(2 ; 13)$ translocation in alveolar rhabdomyosarcomas is a more potent transcriptional activator than PAX3. Mol. Cell Biol., 15(3): 1522-1535.

Galili, N., Davis, R.J., Fredericks, W.J., Mukhopadhyay, S., Rauscher, F.J., Emanuel, B.S., Rovera, G., Barr, F.G., 1993. Fusion of a fork head domain gene to PAX3 in the solid tumour alveolar rhabdomyosarcoma. Nat. Genet., 5(3):230-235. [doi:10.1038/ng1193-230]

Golub, T.R., Slonim, D.K., Tamayo, P., 1999. Molecular classification of cancer: class discovery and class prediction by gene expression monitoring. Science, 286(5439): 531-537. [doi:10.1126/science.286.5439.531]

Gurney, J.G., Young, J.L., Roffers, S.D., Smith, M.A., Bunin, G.R., 2005. Soft Tissue Sarcomas. National Cancer Institute, Bethesda, MD, USA.

Houghton, P., Horton, J., Houghton, J., 1991. Drug Sensitivity and Resistance in the Xenograft Model. In: Maurer, H., Ruymann, R., Pochedly, C. (Eds.), Rhabdomyosarcoma and Related Tumors in Children. Boca Raton, FL, CRC, p.187-204.

Koumarianou, A., Karageorgopoulou, S., Machairas, A., Liakakos, T., Chranioti, S., Dimitriadis, G., Misiakos, E.P., 2012. A pleomorphic rhabdomyosarcoma mimicking an inguinal hernia: a case report and review of the literature. Oncology, 5(1):74-79. [doi:10.1159/000336158]

Ladanyi, M., 1995. The emerging molecular genetics of sarcoma translocations. Diagn. Mol. Pathol., 4(3):162-173. [doi:10.1097/00019606-199509000-00003]

Minn, A.Y., Lyden, E.R., Anderson, J.R., Million, L., Arndt, C.A., Brown, K., Hawkins, D.S., Donaldson, S.S., 2010. Early treatment failure in intermediate-risk rhabdomyosarcoma: results from IRS-IV and D9803-a report from the Children's Oncology Group. J. Clin. Oncol., 28(27): 4228-4232. [doi:10.1200/JCO.2010.29.0247]

Newton, W.A.Jr., Gehan, E.A., Webber, B.L., Marsden, H.B., van Unnik, A.J.M., Hamoudi, A.B., Tsokos, M.G., Shimada, H., Harms, D., Schmidt, D., et al., 1995. Classification of rhabdomyosarcomas and related sarcomas. Pathologic aspects and proposal for a new classificationan intergroup rhabdomyosarcoma study. Cancer, 76(6): 1073-1085. [doi:10.1002/1097-0142(19950915)76:6< 1073::AID-CNCR2820760624>3.0.CO;2-L]

Ordonez, N.G., 1998. Desmoplastic small round cell tumor: II: an ultrastructural and immunohistochemical study with emphasis on new immunohistochemical markers. Am. $J$. Surg. Pathol., 22(11):1314-1327. [doi:10.1097/000004 78-199811000-00002]

Pappo, A.S., Shapiro, D.N., Crist, W.M., Maurer, H.M., 1995. Biology and therapy of pediatric rhabdomyosarcoma. $J$. Clin. Oncol., 13(8):2123-2139.

Pappo, A.S., Lyden, E., Breneman, J., Wiener, E., Teot, L., Meza, J., Crist, W., Vietti, T., 2001. Up-front window trial of topotecan in previously untreated children and adolescents with metastatic rhabdomyosarcoma: an intergroup 
rhabdomyosarcoma study. J. Clin. Oncol., 19(1):213-219.

Parham, D.M., Ellison, D.A., 2006. Rhabdomyosarcoma in adults and children: an update. Arch. Pathol. Lab. Med., 130(10):1454-1465.

Sorensen, P.H., Triche, T.J., 1996. Gene fusions encoding chimaeric transcription factors in solid tumours. Semin. Cancer Biol., 7(1):3-14. [doi:10.1006/scbi.1996.0002]

Sorensen, P.H., Lynch, J.C., Qualman, S.J., Tirabosco, R., Lim, J.F., Maurer, H.M., Bridge, J.A., Crist, W.M., Triche, T.J., Barr, F.G., 2002. PAX3-FKHR and PAX7-FKHR gene fusions are prognostic indicators in alveolar rhabdomyosarcoma: a report from the Children's Oncology Group. J. Clin. Oncol., 20(11):2672-2679. [doi:10. 1200/JCO.2002.03.137]

Triche, T.J., 1997. Pathology and Molecular Diagnosis of Pediatric Malignancies. In: Pizzo, P.A., Poplack, D.G. (Eds.), Principles and Practice of Pediatric Oncology, 3rd Ed. Lippincott-Raven Publishers, Philadelphia, p.54-156.

Wexler, L.H., Helman, L.J., 1997. Rhabdomyosarcoma and the Undifferentiated Sarcomas. In: Pizzo, P.A., Poplack, D.G. (Eds.), Principles and Practise of Pediatric Oncology, 3rd Ed. Lippincott-Raven Publishers, Philadelphia, p.238-329.

\section{Journal of Zhejiang University-SCIENCE B (Biomedicine \& Biotechnology)}

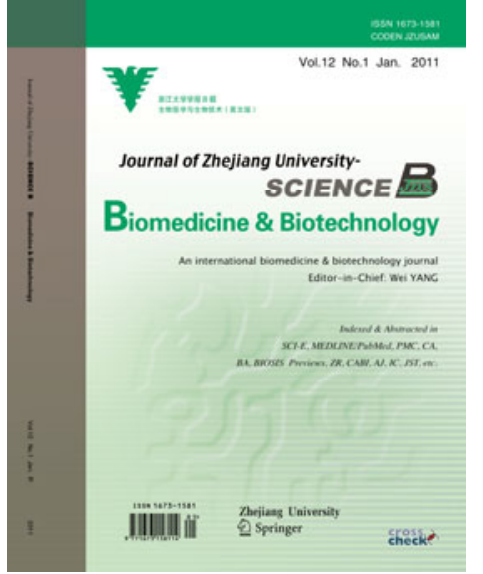

JZUS-B is an international "Biomedicine \& Biotechnology" peer-reviewed journal indexed by SCI-E, MEDLINE, PMC, BA, BIOSIS Previews, JST, ZR, CA, SA, AJ, ZM, CABI, CSA, etc.

It mainly covers Biomedicine, Biochemistry, and Biotechnology, and publishes Research Articles, Science Letters, Communications, New Technologies and so on.

Welcome your interesting contributions!

The Impact Factor in 2012 JCR: J ZHEJIANG UNIV-SC B 1.108 